\title{
Comparative study of anomalous dispersive transparent media
}

\author{
A. Wicht, ${ }^{1, *}$ R.-H. Rinkleff, ${ }^{2}$ L. Spani Molella, ${ }^{1}$ and K. Danzmann ${ }^{1,2}$ \\ ${ }^{1}$ Max-Planck-Institut für Gravitationsphysik, Albert-Einstein-Institut, Teilinstitut Hannover, Callinstrasse 38, D-30167 \\ Hannover, Germany \\ ${ }^{2}$ Institut für Atom- und Molekülphysik, Abteilung Spektroskopie, Universität Hannover, Callinstrasse 38, D-30167 Hannover, Germany
}

(Received 14 May 2002; revised manuscript received 6 September 2002; published 31 December 2002)

\begin{abstract}
We present a comparative theoretical analysis of five different atomic schemes with respect to their performance as negative (anomalous) dispersive transparent media. The schemes discussed are the strongly driven and the degenerate two-level system, two closely spaced gain lines, and two slightly different double- $\Lambda$ schemes with incoherent pumping. The analysis shows that the degenerate two-level scheme is best suited for applications for which only modest negative dispersion but strongly reduced absorption is required, and dissipation and loss of the optical fields have to be avoided. Two closely spaced gain lines and one of the double- $\Lambda$ schemes are the best choice for applications which rely on large negative dispersion, but are not too sensitive to residual absorption and dissipation.
\end{abstract}

DOI: 10.1103/PhysRevA.66.063815

PACS number(s): 42.50.Gy, 42.25.Kb, 32.80.Qk

\section{INTRODUCTION}

Strongly dispersive media have been discussed for a variety of experimental applications. For example, electromagnetically induced transparency (EIT $[1,2]$ ) has been applied to slow down light [3-5] or even to store light in a sample of ultracold atoms [6] or in a vapor cell at room temperature [7]. As this provides nondestructive storage and read out of coherent optical information, much attention is being payed to it within the context of quantum communication and computation ( [7] and references therein). For all of these experiments, strongly positive dispersion and transparency of the medium are essential.

The complementary situation corresponds to strongly negative or anomalous dispersive transparent media, which are the subject of this paper. They have been used to realize superluminal or even negative group velocities [8]. Further, they have been considered for the realization of broadband high-finesse optical cavities [9,10], for which the anomalous dispersion is supposed to cancel the variation of optical wavelength with frequency inside the cavity. These kinds of cavities may be useful for laser interferometric gravitational wave detectors. Moreover, they would provide a true distance measurement between the mirrors of the cavity as the resonance condition would no longer be affected by laser frequency noise. Although both kinds of applications, superluminal pulse propagation and intracavity operation, depend on negative dispersion without absorption, they are subjected to different requirements: to achieve superluminal or even negative group velocities, the negative dispersion should be as large as possible and absorption at the few percentage level is acceptable. For the intracavity application negative dispersion of modest but well-defined amount is required and residual transmission losses have to be suppressed to a level, at which they no longer degrade the finesse of the cavity. In addition, negative dispersive transparent media may be interesting for (intracavity) quantum nondemolition (QND) type

*Electronic address: wicht@pallas.amp.uni-hannover.de of applications, where quantum information on a "signal" beam is transfered to a "meter" beam [11]. In this case dissipation and loss of both the driving (signal) and probing (meter) fields have to be avoided.

The goal of this paper is twofold. First, we will show how three quantities provide the means to determine and compare the performance of different atomic schemes as strongly dispersive transparent media (Sec. II): these are the absorption, the dispersion, and the ratio of dispersion to absorption, which we will call figure of merit. Second, we will discuss and compare all schemes known to the authors to provide negative dispersion without absorption: the strongly driven two-level system (Sec. III), the degenerate two-level-system (Sec. IV), the $\Lambda$ system driven by a bichromatic off-resonant driving field (i.e., two closely spaced gain lines, (Sec. V) and two slightly different double- $\Lambda$ schemes with incoherent pumping (Secs. VI, VII). As one of the results we will show in Sec.VIII that the degenerate two-level system is the best suited for intracavity applications and especially is the only candidate for QND type of measurements. Furthermore, one of the double- $\Lambda$ schemes provides the largest negative dispersion and, followed by the bichromatically driven $\Lambda$ system, therefore is the optimum choice for experiments aiming at superluminal or negative group velocities.

\section{DEFINITION OF TERMS}

We now want to point out the strategy behind our analysis. We will first give a qualitative description of terms used here to determine and compare the performance of different atomic schemes. Then, some general definitions will be presented, which are used throughout the paper to define the different atomic schemes. Finally, at the end of this section, an exact definition of those terms will be given which have been introduced earlier on a qualitative level.

Following the introductory remarks, the figure of merit may be the most important quantity for intracavity type of applications as it determines the amount of dispersion available per residual absorption. However, even ultralarge figures of merit may be of no use if the corresponding disper- 
sion is so small that even at large atomic densities the dispersion of the optical wave number $k$, i.e., $\partial_{\omega} k(\omega)=\{1$ $\left.+[\omega / n(\omega)] \partial_{\omega} n(\omega)\right\} n(\omega) / c_{0}$, barely differs from its vacuum value, which is $\partial_{\omega} k(\omega)=1 / c_{0}$. Here, $n(\omega)$ denotes the index of refraction and $c_{0}$ stands for the vacuum speed of light. Especially for "fast light" applications this dispersion has to be small compared to $1 / c_{0}$ or even has to be negative. Therefore, in order to compare different atomic schemes for different kinds of applications, the dispersion and absorption of a specific system should be plotted in a dispersion vs. absorption graph. Lines of constant ratio of dispersion to absorption then denote those points that provide the same figure of merit. Hence, the dispersion vs. absorption graph is best suited for our discussion.

The most simple system providing negative dispersion is the two-level scheme only interacting with a weak and resonant probe beam. Unfortunately, this is accompanied with strong absorption which is why we have to look for alternative schemes. Any of these will have to provide larger negative dispersion and (or) or larger figures of merit in order to be interesting. Therefore, the simple two-level system interacting only with a weak resonant probe will serve as our reference point and the performance of any other system will be compared against it: a system will be said to provide enhanced dispersion, enhanced absorption, or an enhanced figure of merit, if the corresponding value exceeds the one of the two-level reference system. In this sense, negative dispersion without absorption is achieved if the figure of merit exceeds the reference figure of merit. For sake of clarity, the reference system will be included in all dispersion vs. absorption graphs and will be denoted $P_{A}$. In addition, a line labeled $A$ will be shown in all graphs and will mark all those points which correspond to the figure of merit defined by the reference point $P_{A}$.

Now we want to address the question which schemes to consider. Especially for superluminal (as well as ultraslow) pulse propagation applications, the deformation of the pulse shape due to group velocity dispersion [8] $\partial_{\omega}^{2} n(\omega)$ and dispersion of the absorption $\partial_{\omega} \alpha(\omega)$ should be avoided. Therefore, certain schemes will not be considered here, such as strongly dispersive regions at the Rabi sidebands of the strongly driven two-level system, where the absorption monotonically runs through $\alpha=0\left(\alpha=0\right.$ but $\left.\partial_{\omega} \alpha \neq 0\right)$.

We next turn to the general description of the atomic schemes. The von Neumann equation of a system will, in general, be given by

$$
\imath \hbar \dot{\tilde{\rho}}=\left[\widetilde{\mathcal{H}}_{A}+\widetilde{\mathcal{H}}_{I}, \tilde{\rho}\right]+\imath \hbar \widetilde{\Gamma}(\tilde{\rho})
$$

where $\tilde{\rho}$ is the density matrix describing the state of the system, $\widetilde{\mathcal{H}}_{A}$ is the Hamiltonian of the atom and

$$
\widetilde{\mathcal{H}}_{I}=\sum_{i} d \widetilde{\mathcal{D}}_{k_{i} l_{i}} a_{i} E_{i} \cos \left(\omega_{i} t\right)
$$

describes the electric dipole interaction between the atom and the optical fields. Here $E_{i}=E_{D}, E_{P}$ and $\omega_{i}=\omega_{D}, \omega_{P}$ are the classical electric-field amplitudes and angular fre- quencies of the driving and probing field, respectively. The sum runs over all pairs $\left|k_{i}\right\rangle,\left|l_{i}\right\rangle$ which are coupled through the optical fields via dipole interaction. $a_{i}$ is a factor of order unity used for normalization purposes and

$$
\widetilde{\mathcal{D}}_{k l}=|k\rangle\langle l|+| l\rangle\langle k|
$$

is the generic dipole operator. As we are aiming at a qualitative rather than exact quantitative comparison of different atomic systems we have assumed in Eq. (2), for sake of simplicity, that all dipole matrix elements involved are identical: $d_{k_{i} l_{i}}=d$. The Rabi frequencies for the driving and probing field will always be defined as

$$
\Omega_{i}=\frac{d E_{i}}{2 \hbar},
$$

where again $i=D, P$. In order to describe spontaneous emission or other coherence decay, incoherent pumping or the effect of finite interaction time we write

$$
\widetilde{\Gamma}(\tilde{\rho})=\widetilde{\Gamma}_{S E}(\tilde{\rho})+\widetilde{\Gamma}_{D E}(\tilde{\rho})+\widetilde{\Gamma}_{I P}(\tilde{\rho})+\widetilde{\Gamma}_{T R}(\tilde{\rho}) .
$$

Here

$$
\widetilde{\Gamma}_{S E}=\sum_{i} \gamma_{i} \widetilde{\Gamma}_{k_{i} l_{i}}
$$

sums up all spontaneous emission processes, and

$$
\begin{aligned}
\widetilde{\Gamma}_{k l}= & |l\rangle\langle l|\langle k|\tilde{\rho}| k\rangle-\frac{1}{2} \sum_{m}\{|m\rangle\langle k|\langle m|\tilde{\rho}| k\rangle+| k\rangle \\
& \times\langle m|\langle k|\widetilde{\rho}| m\rangle\}
\end{aligned}
$$

is the generic description of spontaneous emission from state $|k\rangle$ into state $|l\rangle$. The sum in Eq. (7) describes the depopulation of the $|k\rangle$ and the decay of all coherences between $|k\rangle$ and any other state due to the spontaneous emission from $|k\rangle$. In order to account for additional decoherence, we set

$$
\widetilde{\Gamma}_{D E}=\sum_{i}-\kappa_{k_{i} l_{i}}\left\{\left\langle k_{i}|\tilde{\rho}| l_{i}\right\rangle+\left\langle l_{i}|\tilde{\rho}| k_{i}\right\rangle\right\}
$$

where $\kappa_{k l}$ describes the additional decay rate of the $\langle k|\tilde{\rho}| l\rangle$ coherence. Further, incoherent pumping is understood to transfer population from one state to the other without introducing additional coherence between these states. The corresponding contribution to $\widetilde{\Gamma}$ is a sum over all incoherent pumping processes

$$
\widetilde{\Gamma}_{I P}=\sum_{i} r_{k_{i} l_{i}} \widetilde{\mathcal{R}}_{k_{i} l_{i}},
$$

where

$$
\widetilde{\mathcal{R}}_{k l}=|l\rangle\langle l|\langle k|\tilde{\rho}| k\rangle-\frac{1}{2} \sum_{i}\{|i\rangle\langle k|\langle i|\tilde{\rho}| k\rangle+| k\rangle\langle i|\langle k|\tilde{\rho}| i\rangle\}
$$


is the generic description for incoherent pumping from state $|k\rangle$ to state $|l\rangle$. Please note that this pumping does not introduce additional coherence between $|k\rangle$ and $|l\rangle$, but it causes a decay of all coherences between $|k\rangle$ and any other state. Obviously, the description of incoherent pumping Eq. (10) is the same as of spontaneous emission Eq. (7). Incoherent pumping can be realized experimentally, e.g., by broadband optical fields, as it has been demonstrated, for example, by Zibrov and co-workers [12]. Finally, to model the effect of finite interaction time between the atom and the optical fields, we will assume that the atoms are injected at rate $\gamma_{0}$ into the interaction region and that they are equally distributed among the "ground" states of the atom. In order to maintain a constant number of atoms in the interaction zone, atoms are leaving the interaction zone at rate $\gamma_{0}$ in all states of the system

$$
\widetilde{\Gamma}_{T R}=-\gamma_{0} \tilde{\rho}+\sum_{i=1}^{N_{g}} \frac{\gamma_{0}}{N_{g}}\left|l_{i}\right\rangle\left\langle l_{i}\right| .
$$

Here the sum runs over all ground states of the system. $\gamma_{0}$ plays the role of the transit time limit for the coherence life time.

We solve the resulting density-matrix equation either analytically (for the strongly driven two-level system) or numerically under steady-state conditions and restrict ourselves to the discussion of the weak probe limit, i.e., we solve the density-matrix equations to all orders in the driving-field Rabi frequency $\Omega_{D}$ and to first order in the probe field Rabi frequency $\Omega_{P}$. As we are only interested in a comparison of different schemes and not in absolute numbers we define a dimensionless "susceptibility" for the transition $|k\rangle \leftrightarrow|l\rangle$ with respect to the probe field by

$$
\chi_{k l}(\delta)=\frac{\langle k|\tilde{\rho}(\delta)| l\rangle}{\Omega_{P} / \gamma}
$$

where $\gamma$ is specifically defined for each system and usually denotes the excited state population decay rate. $\langle k|\tilde{\rho}(\delta)| l\rangle$ describes that part of the $\langle k|\widetilde{\rho}| l\rangle$ coherence which oscillates at the probe field frequency, and $\delta$ is the probe detuning from some "resonance" frequency defined independently for each system. The total dispersion of the system with respect to the probe field is then given by

$$
\chi(\delta)=\sum_{i} \chi_{k_{i} l_{i}}(\delta)
$$

where the sum runs over all transitions $\left|k_{i}\right\rangle \leftrightarrow\left|l_{i}\right\rangle$ which are electric dipole allowed. To proceed, we define the dimensionless probe field absorption coefficient $\alpha$, the index of refraction $n$ and the dimensionless dispersion of the index of refraction $n^{\prime}$ by

$$
\alpha(\delta)=\Im\{\chi(\delta)\}, \quad n(\delta)=\Re\{\chi(\delta)\}, \quad n^{\prime}(\delta)=\gamma \partial_{\delta} n(\delta) .
$$

We use the convention that $\alpha<0$ corresponds to absorption. The last quantity to define is the figure of merit

$$
\theta_{F O M}=\left|\frac{n^{\prime}(\delta=0)}{\alpha(\delta=0)}\right|
$$

again a dimensionless quantity.

As we will show next, the definitions given above express the absorption, the index of refraction and the dispersion (of the index of refraction) in natural units of the atom. For the most simple system, our reference system, i.e., the two-level atom (TLA) interacting with a single weak probe field, we find $\alpha(\delta)=\frac{1}{2}\left[(\delta / \gamma)^{2}+\frac{1}{4}\right]^{-1}$ and $n^{\prime}(\delta)=-\left[\frac{1}{4}\right.$ $\left.-(\delta / \gamma)^{2}\right] /\left[\frac{1}{4}+(\delta / \gamma)^{2}\right]^{2}$, where $\delta$ is the angular frequency detuning of the probe field from atomic resonance. Therefore, at probe resonance $\delta=0$ we find $\alpha=2, n^{\prime}=-4$, and $\theta_{F O M}=2$ so that the absorption, the dispersion, and the figure of merit are basically of order unity for our reference system. It follows that enhanced absorption (or gain) corresponds to $|\alpha| \gg 2$, and enhanced dispersion is realized, if $\left|n^{\prime}\right| \gg 4$. Further, media can be considered to provide strong dispersion without absorption, if $\theta_{F O M} \gg 2$. The reference two-level atom with a single probe field corresponds to point $P_{A}$ in all dispersion $n^{\prime}$ vs. absorption $\alpha$ graphs, see for example Fig. 8. Points in these graphs corresponding to the figure of merit defined by $P_{A}$, i.e. $\left|n^{\prime}\right|=|2 \alpha|$, will always be shown as curve $A$ to define a reference for the figure of merit.

Finally, we would like to mention that we do not consider inhomogeneous broadening (e.g. Doppler broadening) for sake of simplicity, although this may give rise to interesting consequences [13]. We feel that this topic is beyond the scope of this paper. Also, neglecting inhomogeneous broadening does not constitute any serious experimental constrains as experiments can be performed on atomic beams [10] or cold atomic ensembles [6].

\section{STRONGLY DRIVEN TWO-LEVEL ATOM}

The TLA strongly driven by an optical field has been extensively investigated with the studies being initiated by the work of Rautian and Sobel'man [14] and Mollow [15] and being confirmed experimentally shortly thereafter [16]. For resonant driving the spectra were investigated within the context of negative dispersive transparent media by Szymanowski and co-workers [18], whom the reader is referred to for a more detailed reference to work related to strongly driven TLAs.

A schematic description of the strongly driven TLA is given in Fig. 1. A strong resonant field with Rabi frequency $\Omega_{D}$ is driving the transition $|0\rangle \rightarrow|2\rangle$. A second weak field is probing the spectrum with Rabi frequency $\Omega_{P}$ and angular frequency detuning $\delta=\omega_{P}-\omega_{02}$, where $\omega_{P}$ is the probe laser frequency and $\hbar \omega_{02}$ is the energy separation between the upper and lower level of the atom. The population of the excited state $|2\rangle$ radiatively decays into the ground state $|0\rangle$ at rate $\gamma$, which is not shown in Fig. 1. The interaction Hamiltonian reads

$$
\widetilde{\mathcal{H}}_{I}=d \widetilde{\mathcal{D}}_{02}\left\{E_{D} \cos \left(\omega_{D} t\right)+E_{P} \cos \left(\omega_{P} t\right)\right\},
$$




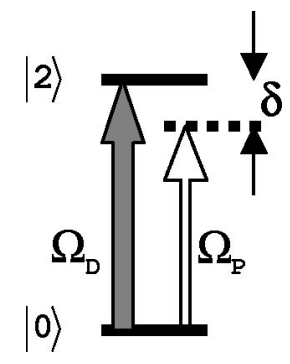

FIG. 1. Strongly driven two-level atom (TLA). A strong resonant driving field with Rabi frequency $\Omega_{D}$ is driving the transition $|0\rangle \rightarrow|2\rangle$. A second weak field with Rabi frequency $\Omega_{P}$ is probing the spectrum with detuning $\delta$ from the atomic resonance.

and the spontaneous emission is given by

$$
\widetilde{\Gamma}_{S E}=\gamma \widetilde{\Gamma}_{20} .
$$

As the population and coherence decay rate of this system is large (on the order of $\gamma$ ), we neglect any additional decoherence due to, e.g., the finite time needed by the atoms to travel through the interaction region. The susceptibility to the probe field is $\chi=\chi_{02}$ [Eq.(12)]. For the solution of the corresponding density-matrix equations the reader is referred to $[17,18]$. This solution shows that in the vicinity of the atomic resonance $(|\delta|<\gamma)$ the probe field absorption is strongly suppressed, while significant negative dispersion still is maintained. At atomic resonance, in the strong driving $\left(\Omega_{D} \gg \gamma\right)$ and weak probing limit $\left(\Omega_{P} \ll \Omega_{D}\right)$, the probe field absorption and dispersion are given by

$$
\begin{gathered}
\alpha=\frac{2}{\left[1+8\left(\Omega_{D} / \gamma\right)^{2}\right]^{2}} \approx \frac{1}{32\left(\Omega_{D} / \gamma\right)^{4}}, \\
n^{\prime}=-\frac{4\left[1+32\left(\Omega_{D} / \gamma\right)^{4}\right]}{\left[1+8\left(\Omega_{D} / \gamma\right)^{2}\right]^{3}} \approx-\frac{1}{4\left(\Omega_{D} / \gamma\right)^{2}} .
\end{gathered}
$$

Hence, we find two basic results: (i) this system provides negative dispersion and (ii) at large driving field intensities, the figure of merit $\Theta_{F O M}=\left|n^{\prime} / \alpha\right|$ increases like $\left(\Omega_{D} / \gamma\right)^{2}$. Therefore, strongly driven two-level atoms can be used to realize negative dispersive transparent media, which has been shown experimentally by our group [19]. However, as $\left|n^{\prime}\right|<4$, enhanced dispersion is not provided. Dispersion vs. absorption for this system is shown in Fig. 2, graph $(C)$.

\section{DEGENERATE TWO-LEVEL SYSTEM}

The degenerate two-level system has been discussed by Friedmann and Wilson-Gordon [20] within the context of enhancing the index of refraction while simultaneously maintaining transparency. They pointed out that this system provides the important feature of neither absorbing the probe nor absorbing the pump field. As the atom's susceptibility to the probe field strongly depends on the pump field Rabi frequency $\Omega_{D}$, this is an important feature in experimental terms because $\Omega_{D}$ must not change along the path of propagation through the medium. Even more importantly, as dis-

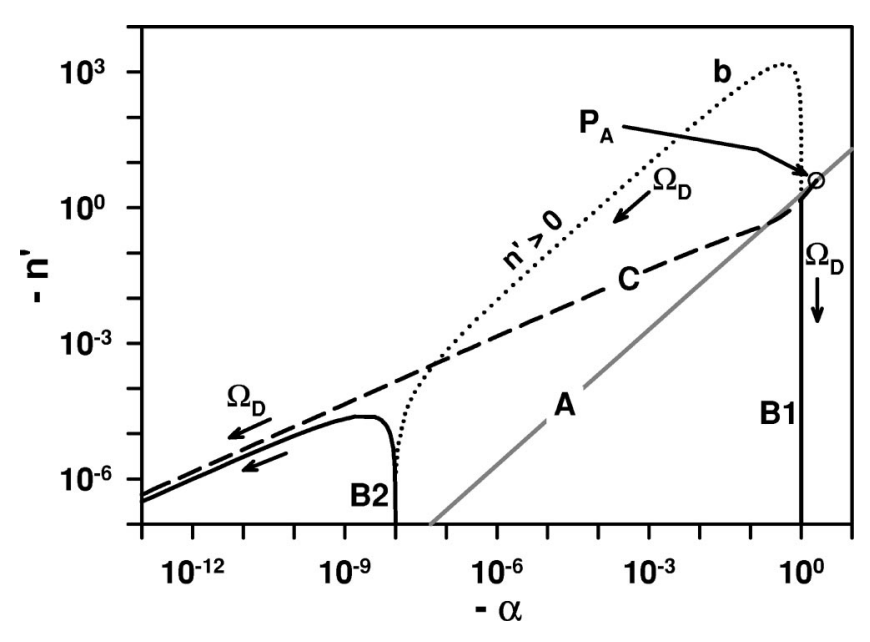

FIG. 2. Performance of the strongly driven TLA and the degenerate TLA: dispersion vs absorption at probe resonance $\delta=0$ with $\Omega_{D}$ varied from $\Omega_{D}=0$ to $\Omega_{D} \gg \gamma$. The arrows indicate the direction of increasing $\Omega_{D} . P_{A}$ TLA-reference point. (A) TLAreference line. $(B 1),(B 2)$ and $(b)$ : degenerate TLA with $\gamma_{0}$ $=10^{-4} \gamma$. For $(B 1),(b)$ and $(B 2)$ the driving field Rabi frequency is $\Omega_{D}<\gamma_{0}, \gamma_{0}<\Omega_{D}<\gamma / \sqrt{2}$ and $\gamma / \sqrt{2}<\Omega_{D}$, respectively. (b) denotes a regime of positive dispersion. $(C)$ strongly driven TLA.

sipation and loss of both the pump and the probe field is avoided, this system should exhibit good signal-to-noise properties at the quantum noise level and may be a candidate for QND type of experiments based on the strong coupling between probe and pump field through the medium.

More recently, Akulshin and co-workers [21] have extensively studied the degenerate two-level system and the dependence of its optical properties on the system parameters. They showed that depending on the angular momenta of the ground and excited state and for linear and orthogonal pump and probe polarizations, electromagnetically induced transparency (EIT) providing strong positive dispersion and electromagnetically induced absorption (EIA) providing strong negative dispersion can be realized. Whereas the first is accompanied with reduced absorption the latter is not, which is a major drawback with respect to what is considered in this paper. However, the authors cited above did not mention that under very specific conditions a degenerate two-level system can actually provide negative dispersion without rather than with increased absorption.

This degenerate TLA is shown in Fig. 3. A linearly polarized strong pump field with Rabi frequency $\Omega_{D}$ is resonantly driving both the $|0\rangle \rightarrow|2\rangle$ and the $|1\rangle \rightarrow|2\rangle$ transitions. A weak probe field with identical polarization is coupling the same transitions and is scanned across the resonance $\delta$ $=\omega_{P}-\omega_{02}=0$. Atoms in the atomic ground states are injected into the interaction region at total rate $\gamma_{0}$. No additional ground-state decoherence is assumed, so that the ground-state coherence lifetime is given by $1 / \gamma_{0}$. The interaction Hamiltonian reads

$$
\widetilde{\mathcal{H}}_{I}=\left\{d \widetilde{\mathcal{D}}_{02}+d \widetilde{\mathcal{D}}_{12}\right\} \frac{1}{\sqrt{2}}\left\{E_{D} \cos \left(\omega_{D} t\right)+E_{P} \cos \left(\omega_{P} t\right)\right\},
$$




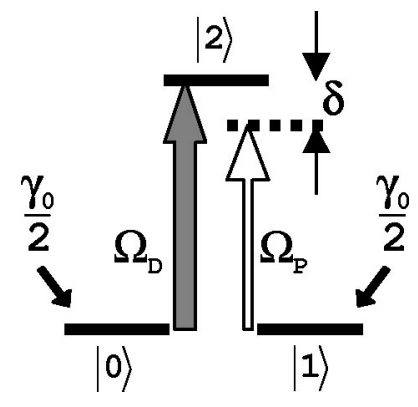

FIG. 3. Degenerate two-level system. A single linearly polarized driving field with Rabi frequency $\Omega_{D}$ is resonantly driving both the $|0\rangle \rightarrow|2\rangle$ and the $|1\rangle \rightarrow|2\rangle$ transitions, where $|0\rangle$ and $|1\rangle$ are degenerate. A weak probe beam with identical polarization also interacts with both transitions and is scanned across the resonance $\delta$ $=\omega_{P}-\omega_{02}$. Atoms are injected at total rate $\gamma_{0}$ into the interaction region.

where $E_{D}$ and $E_{P}$ are the classical electrical-field amplitudes of the linearly and parallel polarized driving and probing field, respectively. Further, the contribution from spontaneous emission is described by

$$
\widetilde{\Gamma}_{S E}=\frac{\gamma}{2}\left(\widetilde{\Gamma}_{20}+\widetilde{\Gamma}_{21}\right),
$$

where $\gamma$ is the population decay rate of the excited state $|2\rangle$ due to spontaneous emission. The injection of atoms into the interaction region is given by

$$
\widetilde{\Gamma}_{T R}=\gamma_{0}\left(-\tilde{\rho}+\frac{1}{2}(|0\rangle\langle 0|+| 1\rangle\langle 1|)\right) .
$$

A system like this could be realized by means of a $F=1$ $\rightarrow F^{\prime}=1$ transition involving the lower-state magnetic sublevels $m= \pm 1$ and the excited-state magnetic sublevel $m^{\prime}$ $=0$. Atoms initially in $|F=1, m=0\rangle$ will be pumped into the $\left|F^{\prime}=1, m^{\prime}= \pm 1\right\rangle$ sublevels, where they eventually decay into the $|F=1, m= \pm 1\rangle$ states. No atoms are lost from $\mid F^{\prime}$ $\left.=1, m^{\prime}=0\right\rangle$ into $|F=1, m=0\rangle$.

We solve the density-matrix equation by the Floquet ansatz described in Ref. [20] to all orders in the driving field Rabi frequency $\Omega_{D}$ and to first order in the probe field Rabi frequency $\Omega_{P}$. Due to the definition of the interaction Hamiltonian in Eq. (20), of the susceptibility in Eq. (12), and of the Rabi frequencies in Eq. (4), the total susceptibility to the probe field is given by $\chi=\sqrt{2}\left(\chi_{02}+\chi_{12}\right)$. The only system parameters to optimize are $\Omega_{D}$ and the ground-state coherence decay rate $\gamma_{0}$.

Our calculations show that for $\Omega_{D} \ll \gamma_{0}$ no significant ground-state coherence is generated and the system basically performs like the two-level reference system. This is shown in Fig. 2, where the probe field dispersion and absorption at probe resonance $\delta=0$ are plotted for the ground-state coherence decay rate $\gamma_{0}=10^{-4} \gamma$ and for the driving field Rabi frequency $\Omega_{D}$ varied from $\Omega_{D}=0$ to $\Omega_{D} \gg \gamma$. For very weak driving $\Omega_{D} \ll \gamma_{0}$ the degenerate TLA is represented by the upper most points of graph $B 1$, which are close to the reference point $P_{A}$. However, if $\Omega_{D}$ is increased beyond $\Omega_{D}$ $=\gamma_{0}$ the situation changes: coherent population trapping (CPT) is established and the system exhibits enhanced positive dispersion, which is shown by graph $b$. As we keep increasing the driving field Rabi frequency beyond $\Omega_{D}$ $=\gamma / \sqrt{2}$ into the strong driving regime the dispersion flips sign again and becomes negative again. This regime, which is represented by graph $B 2$, has three important features. First, our calculation shows that CPT is still maintained so that spontaneous emission from $|2\rangle$ is avoided and neither the probe nor the driving field experience absorption (in the limit of $\left.\gamma_{0} \rightarrow 0\right)$. It has been mentioned before that this is important within the context of QND type of experiments. Please note that the degenerate two-level system is the only negative dispersive system discussed in this paper and known to the authors which provides this feature. Second, the figure of merit $\Theta_{F O M}=\left|n^{\prime} / \alpha\right|$ grows like $\Omega_{D}^{2}$, which is due to the fact that the absorption decreases faster than the dispersion as $\Omega_{D}$ increases (see Fig. 2). This defines the systems ability to provide negative dispersion without absorption. However, as shown in Fig. 2, the value of $\left|n^{\prime}\right|$ is smaller than the reference value defined by $P_{A}$ for any given value of $\Omega_{D}$ within the anomalous dispersive regime. Therefore, enhanced negative dispersion is not accessible. Third, for strong driving $\Omega_{D} \gg \gamma$ the dispersion and the figure of merit provided by the strongly driven TLA can basically also be provided by the degenerate TLA (compare graphs $B 2$ and $C$ ), but then only require much smaller driving field Rabi frequencies as our calculation shows. In fact, the point of maximum dispersion of graph $B 2$ corresponds to $\Omega_{D} \approx \gamma$. Hence large figures of merit can already be achieved at modest driving with the degenerate TLA.

Finally, we want to come back to the fact that in the strong driving regime the degenerate TLA and the strongly driven TLA show the same behavior in the dispersion vs. absorption graph. This can be understood intuitively [20], as the coherent dark state is decoupled from the driving field while the coherent bright state, the excited state, and the driving field are effectively forming a strongly driven twolevel system.

\section{V. $\Lambda$ SYSTEM}

The $\Lambda$ system has been employed by Dogariu and coworkers to propagate an optical pulse at superluminal or even negative group velocities [8], which result from negative dispersion as mentioned in the introduction. These experiments were inspired by the discussion of Steinberg and Chiao [22] who showed theoretically that a gain doublet may be used to realize negative dispersive transparent media. In the work of Dogariu and co-workers a gain doublet was implemented by means of the gain due to two closely spaced Raman transitions in a $\Lambda$-type atomic system. This is shown in Fig. 4. Two driving fields, with frequency difference $2 \Delta_{0}$ and with equal Rabi frequencies $\Omega_{D}$, are strongly detuned from the single-photon resonance of the $|1\rangle \rightarrow|2\rangle$ transition: $\Delta \gg \Omega_{D}, \Delta_{0}, \gamma$. Again, $\gamma$ is the excited state $|2\rangle$ population decay rate due to spontaneous emission. A weak field is probing the $|0\rangle \rightarrow|2\rangle$ transition in the vicinity of the "mean two-photon resonance": $\delta=\omega_{P}-\left(\omega_{02}+\Delta\right)$, where $\Delta$ 


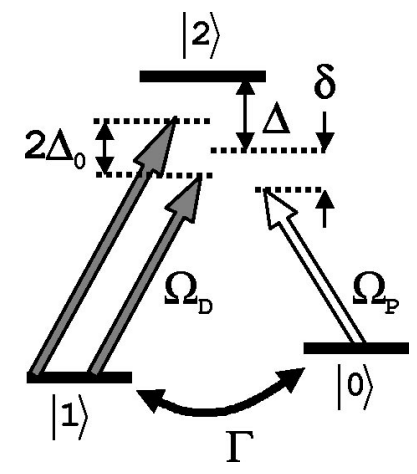

FIG. 4. $\Lambda$ system driven by a bichromatic $\left(\Delta \omega=2 \Delta_{0}\right)$ far offresonant $(\Delta)$ field with the Rabi frequency of both components denoted by $\Omega_{D}$. The monochromatic probe is tuned across the mean of both two-photon resonances. All atoms are pumped into state $|1\rangle$ prior to interaction with the optical fields. Phenomenologically, the ground-state decoherence rate $\Gamma$ has been included.

$=\frac{1}{2}\left(\omega_{D 1}+\omega_{D 2}-2 \omega_{12}\right), \hbar \omega_{02}$ and $\hbar \omega_{12}$ are the energy separations between the lower states and the excited state, and $\omega_{D 1}, \omega_{D 2}$ and $\omega_{P}$ are the driving fields and probing field angular frequencies, respectively. The basic idea is to suppress any absorption related to a single-photon transition by strongly detuning all optical fields from their respective single-photon resonances. Further, in order to actually achieve substantial negative dispersion (and gain) for the probe field, the system has to be prepared into the $|1\rangle$ ground state prior to interaction with the optical fields. The interaction time and the probe and driving field intensities have to be small enough to avoid any substantial population of the states $|0\rangle$ and $|2\rangle$, as in the steady-state case the two-photon population inversion between the ground states will vanish and the sample will basically be "transparent" not only with respect to the single photon but also with respect to the twophoton interaction.

In order to find an expression for the absorption and dispersion of the probe field, we will not make use of Eqs. (1-11), but start from the discussion given by Dogariu and co-workers (Eqs.(24) and (26) in Ref. [8]), who used a simplified solution of the Schrödinger equation to describe this system. They assumed that all atoms are prepared in the $|1\rangle$ state prior to interaction with the driving and probe field. As then state $|0\rangle$ is virtually unpopulated and the probe field amplitude is assumed to be arbitrarily small, any population of the excited state $|2\rangle$ due to the absorption of a probe photon can be neglected. Assuming further (i) that both driving fields are strongly detuned from the single-photon resonance $\Delta \gg \Delta_{0}, \gamma$ and (ii) that the $|1\rangle \rightarrow|2\rangle$ transition is not significantly saturated by the driving field, i.e. $\left(\Omega_{D} / \Delta\right)^{2}$ $\ll \frac{1}{16}$, the absorption and the dispersion of the probe beam at $\delta=0$ are given by

$$
\begin{gathered}
\alpha \approx 2 \frac{\Omega_{D}^{2}}{\Delta^{2}} \frac{\Gamma \gamma}{\Delta_{0}^{2}+\Gamma^{2}}, \\
n^{\prime} \approx-2 \frac{\Omega_{D}^{2}}{\Delta^{2}} \frac{\left(\Delta_{0}^{2}-\Gamma^{2}\right) \gamma^{2}}{\left(\Delta_{0}^{2}+\Gamma^{2}\right)^{2}} .
\end{gathered}
$$

Here, $\Gamma$ has been introduced in order to phenomenologically account for finite ground-state coherence life time. Obviously, the probe beam will always experience gain with the dispersion switching sign at and being negative for the driving field frequency difference obeying $\Delta_{0}>\Gamma$.

If the driving field is increased some atoms will actually be transfered to the excited state. Even, if the single-photon scattering rate due to the bichromatic driving field $2 \Gamma_{S C}$ $=2 \gamma \Omega_{D}^{2} / \Delta^{2}$ is much smaller than the excited-state population decay rate, it can have a substantial effect on the groundstate coherence as this coherence is diminished by any excitation process. Effectively, the two-photon line width $\Gamma$ will be broadened by half of the sum of the two single-photon scattering rates,

$$
\Gamma=\gamma_{0}+\frac{1}{2} 2 \Gamma_{S C}=\gamma_{0}+\gamma \frac{\Omega_{D}^{2}}{\Delta^{2}},
$$

where $\gamma_{0}$ describes the residual nonzero two-photon line width which is determined, for example, by the finite interaction time or collisional dephasing.

In order to analyze the performance of this system we first determine its figure of merit $\Theta_{F O M}$ as

$$
\Theta_{F O M}=\frac{\Delta_{0}^{2}-\Gamma^{2}}{\Delta_{0}^{2}+\Gamma^{2}} \frac{\gamma}{\Gamma},
$$

which approaches its maximum at $\Delta_{0} \gg \Gamma$ to yield $\Theta_{F O M}^{M A X}$ $=\gamma / \Gamma$. Hence, the $\Lambda$ system gains its advantage over a scheme based on two closely spaced dipole allowed optical transitions from the fact that $\Gamma$ can be smaller than typical optical decay rates $\gamma$ by several orders of magnitude.

One parameter to optimize is the driving field frequency difference $2 \Delta_{0}$. For $\gamma_{0}=10^{-2} \gamma$ and the saturation parameter $\epsilon=\Omega_{D}^{2} / \Delta^{2}=10^{-2}$ we start at large $\Delta_{0}=1000 \gamma$. For large frequency differences the dispersion is small, but so is the absorption, see Eq. (23) and graph $(B)$ of Fig. 5. Please note, that the arrows in this figure indicate the direction of increasing $\Delta_{0}$ and $\epsilon$. As we decrease $\Delta_{0}$, both quantities grow while the figure of merit remains constant, see Eq. (25). As $\Delta_{0}$ reaches $\Gamma$, the dispersion switches from negative to positive according to Eq. (23). This is due to the fact that now both Raman transitions are virtually overlapping and negative dispersion, in general, cannot be achieved within the resonance of a single lorentzian shape gain line. From graph $B$ we also note that at maximum dispersion the figure of merit is still close to its maximum value. Obviously, this is the optimum working point. From the expression for the dispersion in Eq. (23) we find, that this point corresponds to $\Delta_{0}^{O P T}=\sqrt{3} \Gamma$.

We will now optimize the saturation parameter $\epsilon$ $=\left(\Omega_{D} / \Delta\right)^{2}$. Please note that according to Eq. (24) the twophoton line width $\Gamma$, and therefore the optimum driving field difference frequency $2 \Delta_{0}^{O P T}=2 \sqrt{3} \Gamma=2 \sqrt{3}\left(\gamma_{0}+\epsilon_{\gamma}\right)$ depend on the saturation parameter and on the residual two-photon line width $\gamma_{0}$. For the optimization of $\epsilon$ we will assume that $\gamma_{0}$ is fixed while $\Delta_{0}$ is always kept at its optimum value $\Delta_{0}^{O P T}(\epsilon)$. The result is given by graph $C$ of Fig. 5. For small 


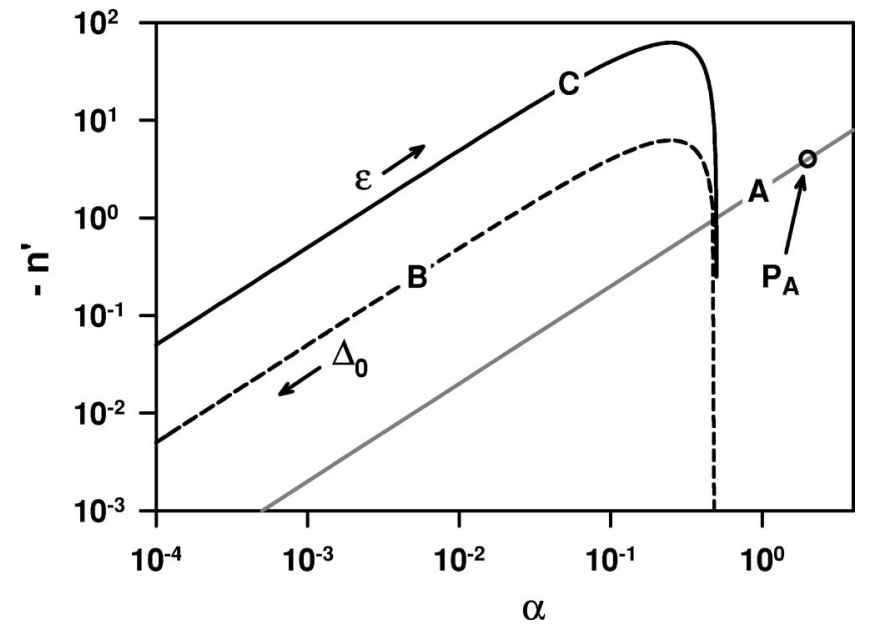

FIG. 5. Performance of the $\Lambda$-system driven by a bichromatic off-resonant field: dispersion vs absorption at probe resonance $\delta$ $=0 . P_{A}$ TLA-reference point. (A) TLA-reference line. (B) $\gamma_{0}$ $=10^{-2} \gamma, \quad \epsilon=10^{-2}, \quad \Delta_{0}=\gamma_{0}, \ldots, 1000 \gamma . \quad(C) \gamma_{0}=10^{-3} \gamma, \epsilon$ $=0, \cdots, 1$ and $\Delta_{0}=\Delta_{0}^{O P T}(\epsilon)=\sqrt{3}, \ldots, \Gamma(\epsilon)$. The arrows associated with graphs $(B)$ and $(C)$ indicate the direction of increasing $\Delta_{0}$ and $\epsilon$, respectively.

saturation parameter $\epsilon$ the two-photon line width $\Gamma$, and hence the optimum driving field difference frequency $2 \Delta_{0}^{O P T}=\Gamma \approx 2 \sqrt{3} \gamma_{0}$ do not depend on $\epsilon$ according to Eq. (24). Therefore the figure of merit $\Theta_{F O M}$ is constant according to Eq. (25), while $\alpha$ and $-n^{\prime}$ both increase linearly with increasing $\epsilon$, see Eq. (23). As the saturation reaches $\epsilon$ $=\left(\Omega_{D} / \Delta\right)^{2}=\gamma_{0} / \gamma$, the two-photon line width is broadened and the figure of merit now starts to decrease as is shown by graph $C$ of Fig. 5. Basically, this means that $\left(\Omega_{D} / \Delta\right)^{2}$ $=\gamma_{0} / \gamma$ is the optimum saturation parameter as it roughly maximizes both the absolute value of the dispersion and the figure of merit simultaneously.

For given $\gamma_{0}$ the optimum system parameters are $\epsilon$ $=\left(\Omega_{D} / \Delta\right)^{2}=\gamma_{0} / \gamma$ and $\Delta_{0}=2 \sqrt{3} \gamma_{0}$. For these parameters, $n^{\prime}=-\gamma /\left(16 \gamma_{0}\right), \alpha=-1 / 4$, and $\Theta_{F O M}^{O P T}=\gamma /\left(4 \gamma_{0}\right)$. Obviously, the goal is to minimize the residual two-photon line width $\gamma_{0}$ in order to achieve both a large figure of merit and large absolute value for the dispersion. Then, the bichromatically driven $\Lambda$ system provides an enhanced figure of merit (for $\gamma_{0}<\gamma / 4$ ) and for $\gamma_{0}<10^{-2} \gamma$ it also provides an enhanced negative dispersion.

Finally, it should be mentioned that negative dispersion without absorption can also be realized with a different kind of $\Lambda$-type system, which has frequently been discussed within the context of EIT and strongly positive dispersive transparent media $[1,2]$. For a single single-photon resonant driving field and a single-photon resonant probe field negative dispersion is provided, only as long as $\Omega_{P}$ exceeds $\Omega_{D}$. Therefore, this system cannot be used in the small signal limit, which is why it has been omitted from our discussion.

\section{DOUBLE- $\Lambda$-SYSTEM I}

The double- $\Lambda$ system shown in Fig. 6 has been studied within the context of lasing without inversion [12], the en-

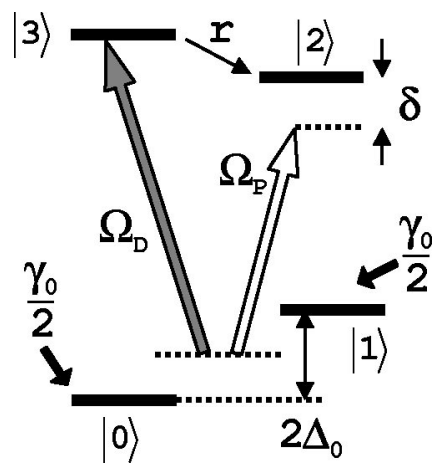

FIG. 6. Double- $\Lambda$ system. The two ground states $|0\rangle$ and $|1\rangle$ are connected to the state $|3\rangle$ through a driving field with Rabi frequency $\Omega_{D}$ tuned to the center of the two single-photon resonances. A weak field with Rabi frequency $\Omega_{P}$, coupling $|0\rangle$ and $|1\rangle$ to state $|2\rangle$ is probing the system. Atoms in state $|0\rangle$ and $|1\rangle$ are injected into the interaction region at rate $\gamma_{0} / 2$ each. Atoms in the excited state $|3\rangle$ are incoherently pumped into $|2\rangle$ at rate $r$. Dephasing of the ground-state coherence at rate $\kappa_{01}=10^{-3}$ has been introduced but is not shown here. For bidirectional incoherent pumping a second rate $r$ (arrow) with direction from the state $|2\rangle$ to the state $|3\rangle$ has to be added.

hancement of the index of refraction [23], and it has also been considered for the realization of negative dispersion without absorption in order to provide broadband high finesse optical cavities [9]. As shown in Fig. 6 each of the ground states is coupled to the "excited driving" level $|3\rangle$ via a single coupling field with Rabi frequency $\Omega_{D}$ and angular frequency $\omega_{D}$, and to the "excited probe" level $|2\rangle$ via a weak probe field with Rabi frequency $\Omega_{P}$ and angular frequency $\omega_{P}$ :

$$
\begin{aligned}
\widetilde{\mathcal{H}}_{I}= & d\left\{\widetilde{\mathcal{D}}_{02}+\widetilde{\mathcal{D}}_{12}\right\} E_{P} \cos \left(\omega_{P} t\right) \\
& +d\left\{\widetilde{\mathcal{D}}_{03}+\widetilde{\mathcal{D}}_{13}\right\} E_{D} \cos \left(\omega_{D} t\right) .
\end{aligned}
$$

The driving field is tuned exactly to the mean of its singlephoton resonances, i.e. $\omega_{D}=\frac{1}{2}\left(\omega_{30}+\omega_{31}\right)$, where $\hbar \omega_{i j}$ is the energy difference between states $|i\rangle$ and $|j\rangle$. The probe field is tuned across its respective mean single-photon resonances, i.e. $\omega_{P}=\frac{1}{2}\left(\omega_{20}+\omega_{21}\right)+\delta$. Probe field "resonance" then is understood to correspond to $\delta=0$. From each of the excited states $|2\rangle$ and $|3\rangle$ the population decays at rate $\gamma / 2$ into each of the ground states $|0\rangle$ and $|1\rangle$ due to spontaneous emission,

$$
\widetilde{\Gamma}_{S E}=\frac{\gamma}{2}\left(\widetilde{\Gamma}_{20}+\widetilde{\Gamma}_{21}+\widetilde{\Gamma}_{30}+\widetilde{\Gamma}_{31}\right)
$$

Hence, $1 / \gamma$ is the population lifetime of both of the excited states. Further, additional decoherence of the ground states

$$
\widetilde{\Gamma}_{D E}=-\kappa_{01}(\langle 0|\tilde{\rho}| 1\rangle+\langle 1|\tilde{\rho}| 0\rangle)
$$

is included. The performance of the system crucially depends on how the incoherent pumping (see rate $r$, Fig. 6) is implemented which is needed to achieve negative dispersion and transparency. In general, it is favorable not to incoherently 
pump atoms out off the atomic ground states as this would diminish the ground-states coherence and reduce the EIT effect. This is why we chose here to incoherently pump atoms from the excited driving state $|3\rangle$ to the excited probe state $|2\rangle$,

$$
\widetilde{\Gamma}_{I P}=r \widetilde{\mathcal{R}}_{32} .
$$

From the numerical solution of the density-matrix equation the total susceptibility to the probe beam is calculated as $\chi$ $=\chi_{02}+\chi_{12}$.

The idea is the following: For strong driving $\Omega_{D} \gg \Delta_{0}$ significant atomic coherence and coherent population trapping (CPT) of the ground states will be established which will provide strong dispersion without absorption for the probe beam. The small fraction of atoms not being trapped in the dark state will be equally distributed among the excited driving state and the bright linear combination of the ground states. Modest incoherent pumping will then ensure that the population in state $|2\rangle$ basically equals the population in the bright state and the sample is rendered transparent for the probe beam.

Before we proceed to optimize the system parameters for maximum figure of merit or maximum negative dispersion we will address the question of how to implement unidirectional and bidirectional incoherent pumping. If one used electromagnetic fields in order to pump atoms from $|3\rangle$ directly to $|2\rangle$, the pumping would actually be bidirectional, with the effective population transfer depending on the population difference between $|3\rangle$ and $|2\rangle$. This method was demonstrated by Zibrov et al. [12], who used a broadband optical field in order to ensure an incoherent transfer of atoms. Of course, for the scheme discussed in this paper, one would have to apply, e.g., a broadband rf-magnetic field to transfer atoms from $|3\rangle$ to $|2\rangle$. Unidirectional incoherent pumping could be realized, for example, via an intermediate exited state $|i\rangle$. Then, atoms could be pumped from $|3\rangle$ to the intermediate state $|i\rangle$ by means of a strong optical field. If a significant fraction of the population in state $|i\rangle$ decays to state $|2\rangle$ at a rate considerably larger than the rate of pumping from $|3\rangle$ to the intermediate state $|i\rangle$, no population is accumulated in $|i\rangle$ and both the "optical pumping" from $|3\rangle$ to $|i\rangle$ and the decay from $|i\rangle$ to $|2\rangle$ are unidirectional.

We next want to optimize three system parameters for the double- $\Lambda$ system with unidirectional pumping: the groundstates energy difference $2 \hbar \Delta_{0}$, the driving field Rabi frequency $\Omega_{D}$ and the incoherent pumping rate $r$. We choose the following strategy: for any given $\Delta_{0}$ and $\Omega_{D}$, the incoherent pumping $r$ is chosen so that the medium becomes transparent for the probe at zero probe detuning $\delta=0$. Then, at fixed $\Delta_{0}$ the driving field Rabi frequency $\Omega_{D}$ is varied and the dispersion at probe resonance $\delta=0$ is calculated. As now the medium is exactly transparent at $\delta=0$, we have to find a different way to quantify how well the absorption is suppressed for a given system. To do so, consider some signal propagating through the strongly dispersive medium. To carry any information, it has to have a certain bandwidth. As the absorption depends on the probe detuning like $\alpha \propto \delta^{2}$ (we have just canceled the constant term, there is no linear term

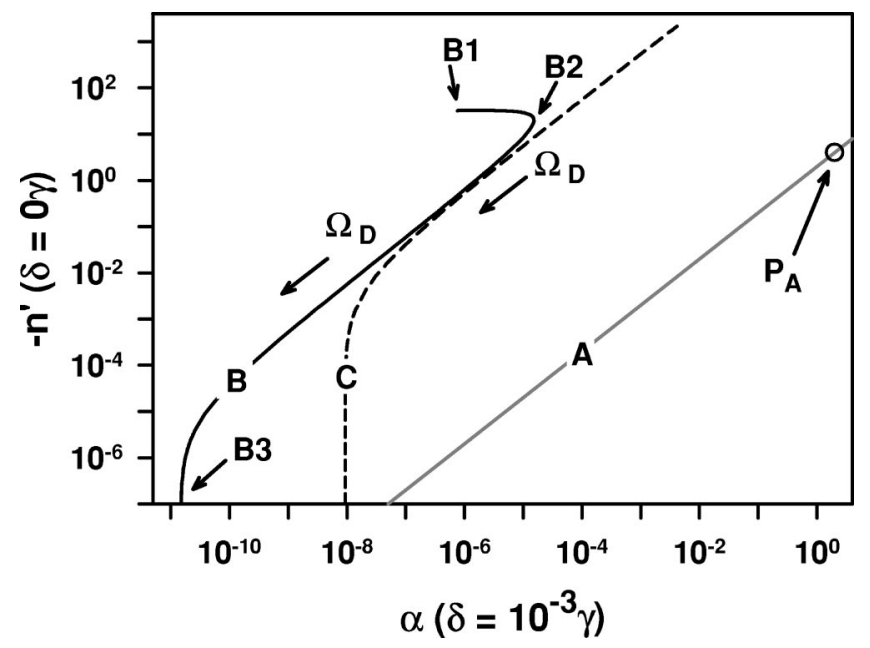

FIG. 7. Performance of the double- $\Lambda$ system with unidirectional incoherent pumping. Dispersion vs absorption for different values of $\Delta_{0}$ with $\Omega_{D}$ varied. For each pair $\left(\Delta_{0}, \Omega_{D}\right)$ the pumping rate $r$ is adjusted to achieve transparency at $\delta=0$. Dispersion and absorption are given at $\delta=0$ and $\delta=10^{-3} \gamma$, respectively. Note: $\alpha \propto \delta^{2}$ for small $\delta . P_{A}$ TLA-reference point. (A) TLA-reference line. $(B)$ $2 \Delta_{0} / \gamma=25, \quad \Omega_{D} / \gamma=3.61, \ldots, 694 \quad$ with $\quad r_{O P T}\left(\Omega_{D}\right) / \gamma$ $=24.8, \ldots, 0.48$. (C) $2 \Delta_{0} / \gamma=1, \Omega_{D} / \gamma=1.27, \ldots, 27.7$ with $r_{O P T}\left(\Omega_{D}\right) / \gamma=2.52, \ldots, 0.48 . B_{1}, B_{2}, B_{3}$ see text. The arrows associated with graphs $(B)$ and $(C)$ indicate the direction of increasing $\Omega_{D}$.

because of the symmetry of the spectra) the absorption at a detuning $\delta$ corresponding to the spectral width of the signal will be a typical value for the residual absorption. As we are not considering a specific signal here, we arbitrarily pick $\delta$ $=10^{-3} \gamma$ and keep in mind that the residual absorption scales like the square of the signal bandwidth, and therefore the figure of merit scales like $\Theta_{F O M} \propto 1 / \delta^{2}$.

The result of our optimization is shown in Fig. 7 for the specific case of $2 \Delta_{0}=25 \gamma$ and $2 \Delta_{0}=\gamma$, see graphs $(B)$ and (C), respectively. It is assumed that $\Delta_{0} \gg \kappa_{0}=10^{-3} \gamma \gg \gamma_{0}$ $=10^{-8} \gamma$. We first want to give a short description of graph $(B)$ and then we will provide a qualitative explanation for the existence of its two different regimes.

Our calculation shows that for the system in order to achieve transparency, $\Omega_{D}$ has to exceed $\Omega_{D}^{2} / \gamma^{2}=\Delta_{0} / \gamma$. This situation corresponds to point $(B 1)$. Increasing $\Omega_{D}$ to $\Omega_{D}$ $=\Delta_{0}$ [point $\left.(B 2)\right]$ actually decreases the figure of merit. If $\Omega_{D}$ is increased beyond $\Omega_{D}=\Delta_{0}$ absorption $\alpha$ and dispersion $-n^{\prime}$ are reduced, while the figure of merit $\Theta_{F O M}$ is maintained. Finally, at large driving field Rabi frequencies $\Omega_{D}^{2} / \Delta_{0}^{2}=\gamma /\left(2 \kappa_{01}\right)$ [point (B3)] the dispersion switches sign and the medium shows normal dispersion without absorption.

For a qualitative understanding of the existence of these different regimes let us first consider arbitrarily small driving field Rabi frequencies. In this case transparency at zeroprobe detuning $\delta=0$ cannot be achieved for any given incoherent pumping rate $r$, which is obvious for $\Omega_{D}=0$. No significant ground-state coherence is generated for $\Omega_{D} \ll \Delta_{0}$, and the behavior of the system can be well understood in terms of a simple rate equation model: we start at $\Omega_{D}, r$ 
$\ll \gamma$ and increase $r$. This reduces the probe absorption, which is expected because population is more efficiently pumped from $|3\rangle$ to $|2\rangle$ thus reducing the $|0\rangle \leftrightarrow|2\rangle$ and $|1\rangle \leftrightarrow|2\rangle$ population difference. However, increasing $r$ even beyond $r$ $=\gamma$ keeps reducing the probe absorption significantly. This is due to the fact that the strong incoherent pumping $r \gg \gamma$ broadens the line width of the driven optical transitions. Due to this broadening the driving field, which is detuned from the single-photon resonances, more effectively pumps atoms from the ground states to the excited state $|3\rangle$. From there, more atoms are transferred to the excited state $|2\rangle$ by incoherent pumping. For strong incoherent pumping $r \approx 2 \Delta_{0}$ $\gg \gamma$ both ground states start to overlap due to the broadening. Increasing $r$ even further, and therefore broadening the driving field transition even more strongly actually starts to reduce the driving field absorption. Hence, now the $|0\rangle \leftrightarrow|2\rangle$ and $|1\rangle \leftrightarrow|2\rangle$ population difference starts to increase. Obviously, for small $\Omega_{D}$ the incoherent pumping that provides the largest reduction of absorption is $r_{O P T}=2 \Delta_{0}$.

Keeping $r=r_{O P T}$ we now increase $\Omega_{D}$ starting at arbitrarily small values. Due to the large detuning $\Delta_{0} \gg \Omega_{D}, \gamma$ of the driving field from the single-photon resonances, neither a significant saturation of the driving transitions is achieved nor a significant ground-state coherence is established, and hence transparency cannot be reached. In order to reach significant saturation a driving field scattering rate has to be established, which is comparable to the radiative decay rate $\gamma$ of $|3\rangle$. For $r_{O P T}=2 \Delta_{0} \gg \gamma$ our calculation shows that this scattering rate is $\Gamma_{S C} \approx \Omega_{D}^{2} / \Delta_{0}$, so that the significant saturation is achieved at $\left(\Omega_{D} / \gamma\right)^{2} \approx \Delta_{0} / \gamma$. Therefore, $\Omega_{D}$ $\approx \sqrt{25 / 2} \gamma$ is the minimum driving field Rabi frequency necessary to achieve transparency at $2 \Delta_{0}=25 \gamma$. This corresponds to point $B 1$ in Fig. 7.

Next we start to increase $\Omega_{D}$ beyond $\Omega_{D}=\sqrt{\Delta_{0} \gamma}$ to $\Omega_{D}$ $\approx \Delta_{0}$, which corresponds to point $B 2$ in Fig. 7 . In this first, incoherent regime the system simply corresponds to two closely spaced gain lines. Enhanced negative dispersion and an enhanced figure of merit are provided at frequencies between the two gain lines.

Now we keep increasing $\Omega_{D}$ beyond $\Omega_{D}=\Delta_{0}$ to $\left(\Omega_{D} / \Delta_{0}\right)^{2}=\gamma /\left(2 \kappa_{01}\right)$, which corresponds to point $B 3$. Please keep in mind that $r$ is always understood to be adjusted to actually achieve transparency at zero probe detuning. Our calculation shows that the incoherent pumping rate $r_{O P T}$ quickly drops from $r_{O P T}=2 \Delta_{0} \gg \gamma$ to $r_{O P T} \approx 0.5 \gamma$ as $\Omega_{D}$ is increased beyond $\Delta_{0}$. Further, significant ground-state coherence is generated. This coherence assists the reduction of probe absorption and - unfortunately - reduces the anomalous dispersion as well. Hence the figure of merit is constant as $\Omega_{D}$ is increasing. In this second, coherent regime negative dispersion is basically restricted to a probe detuning of $|\delta| \leqslant \gamma$.

As we reach $\left(\Omega_{D} / \Delta_{0}\right)^{2}=\gamma /\left(2 \kappa_{01}\right)$ (point B3) the ground states effectively become degenerate due to power broadening of the $|0\rangle \leftrightarrow|3\rangle$ and $|1\rangle \leftrightarrow|3\rangle$ transitions. Beyond this driving level the dispersion flips sign again and becomes positive.
Our detailed calculations aiming at the comparison of the system's performance for various values of the ground-state splitting $2 \Delta_{0}$ show three major results: (i) all parameters provide an enhanced figure of merit whereas only at modest ground-state splitting $\Delta_{0} \sim \gamma$ enhanced dispersion can be realized. Compare graph $(B)$ versus graph $(C)$ of Fig. 7; (ii) within the regime where strong coherence is established, all systems basically provide the same figure of merit; (iii) for $2 \Delta_{0}>\gamma$ the best figure of merit and the largest absolute value for the dispersion are achieved in the incoherent regime, see for example point $B l$ of graph $(B)$. In this regime, already modest driving field Rabi frequencies $\Omega_{D} \geqslant \sqrt{\Delta_{0} \gamma}$ $\gg \gamma$ and large incoherent pumping rates $r \approx 2 \Delta_{0}$ are required and the system qualitatively behaves like two closely spaced gain lines. Our calculations show that large splittings $\Delta_{0}$ are favorable as they guarantee the best figure of merit available at all. As a reminder to the foregoing discussion, we would like to point out again that for the double- $\Lambda$ system with unidirectional pumping the figure of merit, in principle, is limited only by the bandwidth occupied by the probe signal.

We just showed that enhanced dispersion and the largest figures of merit are provided for "modest" driving $\Omega_{D}$ $\sim \Delta_{0}$. However, in this regime a large fraction of the population is accumulated in the excited states $|2\rangle$ and $|3\rangle$. This is an indication for the fact that this parameter regime will not provide good signal-to-noise characteristics for the driving and probe beam at the quantum noise level due to spontaneous emission associated with the exited-state population.

\section{DOUBLE- $\Lambda$-SYSTEM II}

As mentioned in the preceding section, the implementation of incoherent pumping is crucial for the performance of the system in terms of providing negative dispersion without absorption. We next want to show that the bidirectional pumping results in a scheme which does not provide an enhanced figure of merit. The corresponding level scheme is again given by Fig. 6 .

We have modeled the system by essentially using the same Hamiltonian as for the double- $\Lambda$ system with unidirectional pumping but have replaced the incoherent pump contribution by $\widetilde{\Gamma}_{I P}=r\left\{\widetilde{\mathcal{R}}_{23}+\widetilde{\mathcal{R}}_{32}\right\}$. We expect that for this system in general $\rho_{22}<\rho_{33}<\rho_{00}=\rho_{11}$ holds true. Therefore, the medium should not become transparent under any condition (i.e. arbitrary $\Omega_{D}, \Delta_{0}$, and $r$ ), which is confirmed by our simulations.

We now vary the driving field Rabi frequency $\Omega_{D}$ and adjust $r=r_{O P T}\left(\Omega_{D}\right)$ in such a way that the figure of merit is maximized. As an example for this optimization procedure we give the dispersion and the absorption at resonance $\delta$ $=0$ for a ground-state splitting of $\Delta_{0}=\gamma$, see graph $(F)$ of Fig. 8. A comparison of graph $(F)$ versus graph $(A)$ clearly demonstrates that the double- $\Lambda$ system with bidirectional incoherent pumping does not exceed or even reach the figure of merit of the TLA-reference system. Our calculations show that this result holds true for all other ground-state splittings $\Delta_{0} \gg \kappa_{01}$ as well. In this sense, the double- $\Lambda$ system with bidirectional incoherent pumping does not provide negative dispersion without absorption. However, enhanced disper- 


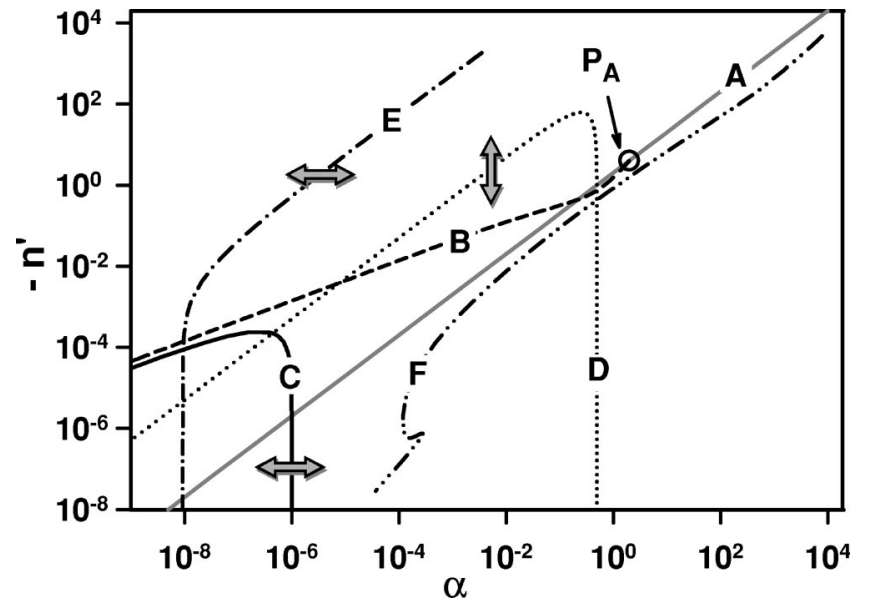

FIG. 8. Comparison of five different atomic schemes with respect to their performance as a negative dispersive transparent medium. The graph shows dispersion vs absorption. $P_{A}$ TLA-reference point. (A) TLA-reference line. $(B)$ Strongly driven TLA, see graph $(C)$ of Fig. 2. $(C)$ Degenerate TLA, see graph $(B)$ of Fig. 2. $(D)$ $\Lambda$-system driven by an off-resonant bichromatic driving field. See graph $(C)$ of Fig. 5. $(E)$ Double- $\Lambda$ system with unidirectional incoherent pumping. See curve $(C)$ of Fig. 7. $(F)$ same as $(E)$, but with bidirectional incoherent pumping rate. Arrows have been added for curves $(C),(D)$, and $(E)$ to indicate the dependence of the performance on individual system parameters. For an explanation see Sec. VIII.

sion and absorption are realized for modest $\Delta_{0}, \Omega_{D} \lesssim \gamma$. The comparison between the double- $\Lambda$ system with unidirectional and bidirectional incoherent pumping clearly shows how strongly the performance of a system does depend on the way incoherent pumping is implemented.

\section{COMPARISON}

In this section we compare the five different schemes. For each of these schemes one graph is shown in Fig. 8: the strongly driven two-level system $(B)$, the degenerate twolevel system $(C)$, the $\Lambda$-scheme driven by a bichromatic off-resonant driving field (the gain doublet) $(D)$, the double$\Lambda$ system with unidirectional incoherent pumping (double$\Lambda$-system I) ( $E$ ), and the double- $\Lambda$ system with bidirectional incoherent pumping (double- $\Lambda$-system II) $(F)$. Again, the reference point defined by the simple two-level system with a weak probe is given as point $P_{A}$ and curve $A$ as usually defines the reference line by indicating all points which provide the same figure of merit as the reference two-level system.

According to Fig. 8 the double- $\Lambda$ system with bidirectional incoherent pumping, graph $(F)$, is the only scheme providing enhanced absorption. The dispersion is also enhanced, but this is less pronounced, so that the figure of merit never exceeds the reference value.

The double- $\Lambda$ system with unidirectional incoherent pumping, graph $(E)$, is the only system providing perfect transparency. In principle, its figure of merit is limited only by the spectral bandwidth $\delta_{S I G}$ of the probe signal: as the absorption is exactly canceled at probe resonance $\delta=0$ we now take the absorption at a detuning equal to half width at half maximum bandwidth of a probe signal as a measure for the residual absorption. Since $\alpha \propto \delta^{2}$, the absorption decreases and the figure of merit $\Theta_{F O M}=\left|n^{\prime} / \alpha\right|$ increases quadratically as $\delta_{S I G}$ decreases. A probe signal bandwidth reduction, say, of a factor of 10 therefore reduces the absorption by a factor of 100 , which moves graph $(E)$ to the left. This has been indicated by an arrow. Furthermore, as we have shown for this system, negative dispersion can be realized over a spectral range which is large compared to $\gamma$, if the ground-state energy spitting is large compared to $\gamma$ and the driving field intensity is modest $\left(\sqrt{\Delta_{0} \gamma}<\Omega_{D}<\Delta_{0}\right)$. This regime also provides the largest negative dispersion and the largest figure of merit among all schemes discussed in this paper. However, there is a major drawback associated with this scheme. For some applications such as broadband high finesse optical cavities [9] the absorption has to be canceled very precisely at zero-probe detuning $\delta=0$ and the negative dispersion very accurately has to match a certain value. Unfortunately, the residual absorption and the dispersion strongly depend on the incoherent pumping rate, which poses serious requests on an experimental realization.

The bichromatically driven $\Lambda$ system $(D)$ provides an enhanced figure of merit if the ground-state coherence decay rate $\gamma_{0}$ is small compared to $\gamma$ and it even shows enhanced negative dispersion if $\gamma_{0} \lesssim 10^{-2} \gamma$. In fact, the whole graph $(D)$ is shifted up or down if $\gamma_{0}$ is decreased or increased, respectively. This has been indicated by an arrow in Fig. 8. The advantage of this system consists in providing its maximum figure of merit and maximum dispersion at driving field Rabi frequencies $\Omega_{D} \ll \gamma$ which can be smaller than the Rabi frequencies $\Omega_{D} \sim \Delta_{0} \geqslant \gamma$ needed for the double- $\Lambda$ system with unidirectional pumping $(E)$ by several orders of magnitude. If very large figures of merit $\left(\Theta_{F O M} \geqslant 10^{6}\right)$ are required for only a small probe signal bandwidth $\delta_{S I G}$ the performance of the bichromatically driven $\Lambda$ system $(D)$ will, however, tend to be inferior to that of the double- $\Lambda$ scheme with unidirectional incoherent pumping $(E)$ : with the bichromatically driven $\Lambda$ system negative dispersion without absorption is achieved over a spectral bandwidth of order $\gamma_{0}$, see Sec. V. As $\gamma_{0}$ has to obey $\gamma_{0}>\delta_{S I G}$ its maximum figure of merit scales like $\gamma / \delta_{S I G}$ [Eq.(25)], whereas it scales like $\left(\gamma / \delta_{S I G}\right)^{2}$ for the double- $\Lambda$ scheme with unidirectional pumping (see Sec. VI). Finally it should be emphasized that the bichromatically driven $\Lambda$ system relies on the following assumptions: all atoms are prepared in a single atomic state prior to the interaction with the driving and probing field and no significant redistribution of atomic population does occur during this interaction. This may again require special experimental attention if one is aiming at very large figures of merit.

The strongly driven two-level system $(B)$ does not provide enhanced dispersion but can be used to realize an enhanced figure of merit. Its advantage consists in not relying on long-living ground-state coherences as opposed to the bichromatically driven $\Lambda$ system. It also does not depend on how well and how precisely incoherent pumping can be established, a fact which is essential for the performance of the 
double- $\Lambda$ system $(E)$. As the figure of merit scales like $\left(\Omega_{D} / \gamma\right)^{2}$ (see Sec. III), large figures of merit can be achieved if powerful lasers are available.

Finally, the degenerate two-level system $(C)$ has some remarkable features. From the comparison with $(B)$, the strongly driven two-level system, we find that both schemes basically show the same dispersion vs. absorption graphs. However, by means of small ground-state decoherence rates, large figures of merit can be achieved with the degenerate two-level system at a modest driving field Rabi frequency $\Omega_{D} \sim \gamma$, as it has been mentioned in Sec. IV. From that section it also follows, that a reduction or increase of the ground-state decoherence rate $\gamma_{0}$ shifts graph $(C)$ to the left or right along graph $(B)$. This has been indicated by an arrow. Please note that maximum dispersion of $(C)$ corresponds to $\Omega_{D} \approx \gamma$. This is a significant advantage over the strongly driven two-level system. Even more important is the fact that the degenerate two-level system is the only scheme providing negative dispersion without absorption while avoiding dissipation of both the driving and probing fields. Therefore this system is the only interesting candidate for QND type of measurements.

Obviously, the optimum choice of the system depends on the application one has in mind. For certain applications, the enhancement of dispersion can be the most essential goal while residual absorption (say, at a few percentage level) is acceptable. Then, the bichromatically driven $\Lambda$ system $(D)$ as well as the double- $\Lambda$ system with unidirectional pumping $(E)$ may be the most promising candidates. This is the case e.g. for experiments on superluminal pulse propagation [8]. However, if the figure of merit is the most essential feature, the strongly driven two-level system $(B)$ or the degenerate two-level system $(C)$ may be the optimum choice. This applies, for example, to the realization of broadband highfinesse optical cavities. If powerful lasers are available, $(B)$ is the most simple scheme to be implemented experimentally, as it does not rely on small ground-states decoherence rates. However, if the dissipation and loss of both the driving and probing fields have to be avoided, the degenerate twolevel system $(C)$ will be the only choice available.

\section{CONCLUSION}

We have shown that three (or equivalently two independent) quantities may be used to describe and compare the performance of different atomic schemes within the context of strongly dispersive transparent media: the absorption, the dispersion (of the index of refraction), and the figure of merit given by the ratio of the dispersion to the absorption. These quantities are always compared to the values corresponding to a simple two-level system interacting with a weak and resonant probe field. With respect to this reference scheme, we define regimes of enhanced or reduced absorption, enhanced dispersion, and enhanced figure of merit. Five different atomic schemes have been discussed: the strongly driven two-level system, a specific degenerate two-level system, a $\Lambda$-system driven by a bichromatic, off-resonant driving field, and a double- $\Lambda$ system with unidirectional and bidirectional incoherent pumping. These are the only schemes not subjected to group velocity dispersion which are known to the authors to provide negative dispersion without absorption or, equivalently, and enhanced figure of merit.

The last scheme mentioned turned out to provide enhanced absorption and dispersion without enhancing the figure of merit. The largest dispersion is provided by the double- $\Lambda$ system with unidirectional incoherent pumping, which also is the only scheme providing perfect transparency. The bichromatically driven $\Lambda$ system is competitive in the regime of modestly enhanced dispersion and figure of merit, as its experimental implementation is more straightforward. However, for intracavity applications of these type of media, the figure of merit is the most crucial parameter and the strongly driven or degenerate two-level system are the most suitable schemes. The degenerate two-level-system has tighter experimental constrains than the strongly driven TLA but shows a remarkable advantage over the latter and all other schemes: it provides an enhanced figure of merit while avoiding dissipation of both the driving and probing field. This is an important feature for QND type of applications. Further, when compared to the strongly driven twolevel scheme, it achieves large figures of merit at smaller driving field Rabi-frequencies $\Omega_{D} \sim \gamma$, which is an important fact in experimental terms. Finally, the degenerate two-levelsystem is likely to provide the largest figure of merit of all schemes which were discussed.

Note added in proof: Recently, Wilson-Gordon and Friedmann [24] discussed the nearly degenerate $\Lambda$ system. In the limit of strong driving (degenerate limit) they find negative dispersion without absorption.

\section{ACKNOWLEDGMENT}

This work was supported by the Grant No. SFB 407 of the Deutsche Forschungsgemeinschaft.
[1] S. E. Harris, Phys. Today 50(7), 36 (1997).

[2] E. Arimondo, Progress in Optics $X X X V$, edited by E. Wolf (Elsevier Science, Amsterdam, 1996), p. 257

[3] L. V. Hau, S. E. Harris, Z. Dutton, and C. H. Behroozi, Nature (London) 397, 594 (1999).

[4] D. Budker, D. F. Kimball, S. M. Rochester, and V. V. Yashchuk; Phys. Rev. Lett. 83, 1767 (1999).

[5] M. M. Kash, V. A. Sautenkov, A. S. Zibrov, L. Hollberg, G. R. Welch, M. D. Lukin, Yu. Rostovtsev, E. S. Fry, and M. O.
Scully, Phys. Rev. Lett. 82, 5229 (1999).

[6] Ch. Liu, Z. Dutton, C. H. Behroozi, and L. V. Hau, Nature (London) 409, 490 (2001).

[7] D. F. Phillips, A. Fleischhauer, A. Mair, R. L. Walsworth, and M. D. Lukin, Phys. Rev. Lett. 86, 783 (2001).

[8] A. Dogariu, A. Kuzmich, and L. J. Wang, Phys. Rev. A 63, 053806 (2001); L. J. Wang, A. Kuzmich, and A. Dogariu, Nature (London) 406, 277 (2000); S. Chu and S. Wong, Phys. Rev. Lett. 48, 738 (1982). 
[9] A. Wicht, K. Danzmann, M. Fleischhauer, M. Scully, G. Müller, and R.-H. Rinkleff, Opt. Commun. 134, 431 (1996).

[10] A. Wicht, M. Müller, R.-H. Rinkleff, A. Rocco, and K. Danzmann, Opt. Commun. 179, 107 (2000).

[11] P. Grangier, J. A. Levenson, and J.-P. Poizat, Nature (London) 396, 537 (1998).

[12] A. S. Zibrov, M. D. Lukin, D. E. Nikonov, L. Hollberg, M. O. Scully, V. L. Velichansky, and H. G. Robinson, Phys. Rev. Lett. 75, 1499 (1995).

[13] O. Kocharovskaya, Y. Rostovtsev, and M. O. Scully, Phys. Rev. Lett. 86, 628 (2001).

[14] S. G. Rautian and I. I. Sobel'man, Zh. Éksp. Teor. Fiz. 41, 456 (1961) [Sov. Phys. JETP 14, 328 (1962)].

[15] B. R. Mollow, Phys. Rev. 188, 1969 (1969).

[16] W. Hartig and H. Walther, Appl. Phys. 1, 171 (1973).

[17] H. Friedmann and A. D. Wilson-Gordon, Phys. Rev. A 36, 1333 (1987).
[18] K. Szymanowski, A. Wicht, and K. Danzmann, J. Mod. Opt. 44, 1373 (1997).

[19] A. Rocco, A. Wicht, R.-H. Rinkleff, and K. Danzmann, Phys. Rev. A (to be published).

[20] H. Friedmann and A. D. Wilson-Gordon, Opt. Commun. 98, 303 (1993).

[21] A. M. Akulshin, S. Barreiro, and A. Lezama, Phys. Rev. Lett. 83, 4277 (1999); A. Lezama, S. Barreiro, A. Lipsich, and A. M. Akulshin, Phys. Rev. A 61, 013801 (1999); A. Lipsich, S. Barreiro, A. M. Akulshin, and A. Lezama, ibid. 61, 053803 (2000);

[22] A. M. Steinberg and R. Y. Chiao, Phys. Rev. A 49, 2071 (1994).

[23] M. Fleischhauer, C. H. Keitel, M. O. Scully, Chang Su, B. T. Ulrich, and Shi-Yao Zhu, Phys. Rev. A 46, 1468 (1992).

[24] A. D. Wilson-Gordon and H. Friedmann, J. Mod. Opt. 49, 125 (2002). 